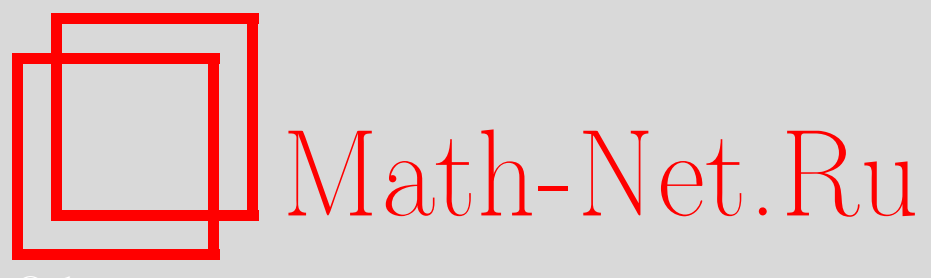

Ю. М. Дюкарев, Примеры блочных матриц Якоби, порождающих симметрические операторы с любыми возможными дефектными числами, Матем. сб., 2010, том 201, номер 12, 83-92

DOI: https://doi.org/10.4213/sm7628

Использование Общероссийского математического портала Math-Net.Ru подразумевает, что вы прочитали и согласны с пользовательским соглашением http: //www . mathnet.ru/rus/agreement

Параметры загрузки:

IP: 3.80 .181 .102

26 апреля 2023 г., 12:21:08

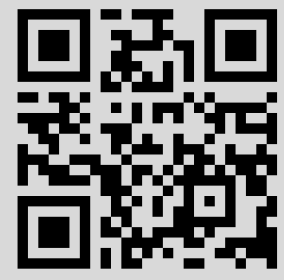


Ю. М. Дюкарев

\section{Примеры блочных матриц Якоби, порождающих симметрические операторы с любыми возможными дефектными числами}

В работе впервые построены примеры симметрических блочных матриц Якоби, порождающих симметрические операторы с произвольными возможными дефектными числами. Приведены явные формулы для базисов в дефектных подпространствах.

Библиография: 7 названий.

Ключевые слова: симметрические блочные матрицы Якоби, симметрические операторы, дефектные подпространства, дефектные числа.

\section{§ 1. Введение}

1.1. Рассмотрим бесконечную симметрическую блочную матрицу Якоби

$$
\mathbf{J}_{m}=\left(\begin{array}{ccccc}
A_{0} & B_{0} & O & O & \ldots \\
B_{0}^{*} & A_{1} & B_{1} & O & \ldots \\
O & B_{1}^{*} & A_{2} & B_{2} & \\
O & O & B_{2}^{*} & A_{3} & \ddots \\
\vdots & \vdots & & \ddots & \ddots
\end{array}\right) .
$$

Блоки матрицы $\mathbf{J}_{m}$ обозначают комплексные квадратные матрицы $m$-го порядка, причем матрицы $A_{k}, k \geqslant 0$, являются эрмитовыми, матрицы $B_{k}, k \geqslant 0,-$ невырожденными, а матрицы $O$ - нулевыми.

Пусть $\mathbb{C}^{m}$ - линейное пространство $m$-мерных столбцов комплексных чисел, а $\ell^{2}\left(\mathbb{C}^{m}\right)$ - гильбертово пространство бесконечных столбцов

$$
\mathbf{u}=\operatorname{col}\left(u_{0}, u_{1}, u_{2}, \ldots\right), \quad u_{k} \in \mathbb{C}^{m}, \quad \sum_{k=0}^{\infty} u_{k}^{*} u_{k}<+\infty
$$

со скалярным произведением $(\mathbf{u}, \mathbf{v})=\sum_{k=0}^{\infty} u_{k}^{*} v_{k}, \mathbf{u}, \mathbf{v} \in \ell^{2}\left(\mathbb{C}^{m}\right)$. На незамкнутом всюду плотном в $\ell^{2}\left(\mathbb{C}^{m}\right)$ подпространстве финитных векторов корректно определен симметрический оператор умножения на матрицу $\mathbf{J}_{m}$. Замыкание этого оператора обозначим через $\mathbf{L}_{m}$. Будем говорить, что симметрический оператор $\mathbf{L}_{m}$ ассоциирован с матрицей Якоби $\mathbf{J}_{m}$. Пусть $\mathbf{I}_{m}$ - тождественный оператор в $\ell^{2}\left(\mathbb{C}^{m}\right)$.

Приведем известные свойства оператора $\mathbf{L}_{m}$ (см. [1]-[3])). Симметрический оператор $\mathbf{L}_{m}$, вообще говоря, не самосопряжен. Подпространства

$$
\mathscr{K}_{+}=\left\{\mathbf{u} \in \ell^{2}\left(\mathbb{C}^{m}\right): \mathbf{L}_{m}^{*} \mathbf{u}=i \mathbf{u}\right\}, \quad \mathscr{K}_{-}=\left\{\mathbf{u} \in \ell^{2}\left(\mathbb{C}^{m}\right): \mathbf{L}_{m}^{*} \mathbf{u}=-i \mathbf{u}\right\}
$$


называются дефектными подпространствами оператора $\mathbf{L}_{m}$ в точках $z=i$ и $z=-i$ соответственно. Векторы из подпространства $\mathscr{K}_{+}$(соответственно $\left.\mathscr{K}_{-}\right)$ называются дефектными векторами оператора $\mathbf{L}_{m}$ в точках $z=i$ и $z=-i$ соответственно. Дефектные числа $m_{+}=\operatorname{dim} \mathscr{K}_{+}$и $m_{-}=\operatorname{dim} \mathscr{K}_{-}$удовлетворяют условиям $0 \leqslant m_{+} \leqslant m, 0 \leqslant m_{-} \leqslant m$ и максимального значения $m$ достигают одновременно (см. [4]). В статье [5] получены некоторые достаточные условия на элементы матрица Якоби (1.1), при выполнении которых дефектные числа $m_{+}$и $m_{-}$максимальны. В статье [6] доказана такая теорема.

Теорема 1. Пусть дано число $m \in \mathbb{N}$ и целье числа $q$, $p$ удовлетворяют условиям $0 \leqslant q, p \leqslant m-1$. Тогда существует такая матрица Якоби $\mathbf{J}_{m}$, что дефектные числа $m_{+}$и $m_{-}$ассочиированного оператора $\mathbf{L}_{m}$ равны $q$ и $p$ соответственно.

1.2. Процитированные результаты дают полное описание возможных значений дефектных чисел операторов $\mathbf{L}_{m}$, порожденных блочными матрицами Якоби $\mathbf{J}_{m}$. Однако задача построения примеров матриц Якоби, порождающих симметрические операторы с произвольными возможными дефектными числами, при этом не была решена.

В настоящей работе впервые построены конкретные примеры блочных матриц Якоби, порождающих симметрические операторы с любыми возможными дефектными числами (см. теоремы 2, 3). Таким образом, в частности, получено новое конструктивное доказательство теоремы 1. Следует отметить, что в работе использованы и впервые развиты в конструктивном направлении методы, предложенные в статье [7].

\section{§ 2. Формулировка основных результатов}

2.1. Пусть $I$ - единичная матрица $m$-го порядка. Диагональную матрицу с элементами $\alpha_{1}, \alpha_{2}, \ldots, \alpha_{m}$ на главной диагонали обозначим через $\operatorname{diag}\left(\alpha_{1}\right.$, $\left.\alpha_{2}, \ldots, \alpha_{m}\right)$.

ТеОрема 2. Пусть целье числа $m \geqslant 1 u p \geqslant 0$ удовлетворяют условию $0 \leqslant p \leqslant m$ и диагональные матрицы $\widehat{B}_{k}$ и $R_{k}$ определены формулами

$$
\begin{gathered}
\widehat{B}_{k}=\operatorname{diag}(\underbrace{\frac{1}{k+1}, \ldots, \frac{1}{k+1}, \underbrace{1, \ldots, 1}_{m-p}), \quad k \geqslant 0,}_{p} \\
R_{0}=I, \quad R_{k}=\sqrt{I+\widehat{B}_{k-1}^{2}}, \quad k \geqslant 1 .
\end{gathered}
$$

Пусть блоки $A_{k}$ и $B_{k}$ матрицъ Якоби $\mathbf{J}_{m}$ имеют следующий вид:

$$
A_{k}=O, \quad k \geqslant 0, \quad B_{0}=\widehat{B}_{0}, \quad B_{k}=\widehat{B}_{k-1}^{-1} R_{k} \widehat{B}_{k}^{-1}, \quad k \geqslant 1 .
$$

Пусть, далее,

$$
\mathbf{P}_{ \pm}=\operatorname{col}\left(( \pm i)^{0} R_{0},( \pm i)^{1} \widehat{B}_{0} R_{0}, \ldots,( \pm i)^{n} \widehat{B}_{n-1} \prod_{k=0}^{n-1} R_{k}, \ldots\right)
$$

Тогда для ассоциированного с матрищей Якоби $\mathbf{J}_{m}$ оператора $\mathbf{L}_{m}$ справедливы следующие утверждения: 
1) первые $р$ столбцов матрицы $\mathbf{P}_{+}$образуют базис в дефектном подпространстве $\mathscr{K}_{+}$, а первые р столбцов матрицы $\mathbf{P}_{-}$образуют базис в дефектном подпространстве $\mathscr{K}_{-}$;

2) дефектные числа $m_{-}$и $m_{+}$оператора $\mathbf{L}_{m}$ совпадают и равны $p$.

ЗАмЕчаниЕ 1. Из (2.1)-(2.3) следует, что

$$
\begin{gathered}
R_{0}=I, \quad R_{k}=\operatorname{diag}\left(\sqrt{1+\frac{1}{k^{2}}}, \ldots, \sqrt{1+\frac{1}{k^{2}}}, \sqrt{2}, \ldots, \sqrt{2}\right), \quad k \geqslant 1, \\
A_{k}=O, \quad k \geqslant 0, \quad B_{0}=I, \\
B_{k}=\operatorname{diag}\left((k+1) \sqrt{k^{2}+1}, \ldots,(k+1) \sqrt{k^{2}+1}, \sqrt{2}, \ldots, \sqrt{2}\right), \quad k \geqslant 1 .
\end{gathered}
$$

Таким образом, операторы с произвольными равными дефектными числами можно построить с помощью простейших блочных матриц Якоби (1.1), у которых все блоки $A_{k}$ являются нулевыми матрицами, а все блоки $B_{k}$ являются положительными диагональными матрицами (2.6).

2.2. Пусть дано целое число $r \geqslant 2$. Рассмотрим матрицу $r$-го порядка

$$
\mathscr{P}_{r}=\left(\begin{array}{cccccc}
0 & 1 & 0 & \ldots & 0 & 0 \\
0 & 0 & 1 & \ldots & 0 & 0 \\
\vdots & \vdots & \vdots & \ddots & \vdots & \vdots \\
0 & 0 & 0 & \ldots & 0 & 1 \\
1 & 0 & 0 & \ldots & 0 & 0
\end{array}\right) .
$$

Для любой матрицы $r$-го порядка $A$ произведение $A \cdot \mathscr{P}_{r}$ получается из матрицы $A$ циклической перестановкой столбцов: $r$-й столбец становится первым, первый столбец становится вторым и т.д., $(r-1)$-й столбец становится $r$-м.

Пусть целые числа $q, p$ и $m$ таковы, что $0 \leqslant q<p<m$. Рассмотрим блочно диагональную матрицу $m$-го порядка

$$
\mathscr{P}=\operatorname{diag}\left(I_{q}, \mathscr{P}_{p-q+1}, I_{m-p-1}\right),
$$

матрица $\mathscr{P}_{p-q+1}$ определена в $(2.7)$, а $I_{q}$ и $I_{m-p-1}$ - единичные матрицы порядка $q$ и $m-p-1$ соответственно. Если нижний индекс у матрицы $I_{q}$ или $I_{m-p-1}$ равен нулю, то в представлении (2.8) соответствующая единичная матрица отсутствует. Для любой матрицы $m$-го порядка $A$ произведение $A \cdot \mathscr{P}_{r}$ получается из матрицы $A$ циклической перестановкой столбцов в диапазоне от $(q+1)$-го столбца до $(p+1)$-го столбца.

Матрица Я尹Р является ортогональной. Поэтому существует вещественное число $\theta$ такое, что корректно определена эрмитова матрица

$$
\mathscr{A}=i\left(I+e^{i \theta \mathscr{P}}\right)^{-1}\left(I-e^{i \theta \mathscr{P}}\right) .
$$

Теорема 3. Пусть иелье числа $q, p$ и m удовлетворяют условиям $0 \leqslant q<$ $p<m$, матрицъ ЯР и $\mathscr{A}$ определенъ формулами (2.8), (2.9), а матрицъ $\widehat{B}_{k}, R_{k}$, $k \geqslant 0$, определены в (2.1), (2.2). Пусть блоки $A_{k}$ и $B_{k}$ матричь Якоби $\mathbf{J}_{m}$ имеют следующий вид:

$$
\begin{aligned}
B_{0} & =\widehat{B}_{0}, \quad B_{k}=2 \widehat{B}_{k-1}^{-1} R_{k}\left(I+e^{i \theta \mathscr{P})^{-1} \widehat{B}_{k}^{-1}, \quad k \geqslant 1,}\right. \\
A_{0}=O, \quad A_{1} & =\widehat{B}_{0}^{-1} R_{1} \mathscr{A} R_{1} \widehat{B}_{0}^{-1}, \quad A_{k}=\widehat{B}_{k-1}^{-1}\left(\mathscr{A}+R_{k} \mathscr{A} R_{k}\right) \widehat{B}_{k-1}^{-1}, \quad k \geqslant 2 .
\end{aligned}
$$


Пусть матрица $\mathbf{P}_{-}$определена в (2.4), а матрица $\mathbf{P}_{+}$задана формулой

$$
\mathbf{P}_{+}=\operatorname{col}\left(i^{0} R_{0} \mathscr{P}_{0}, i^{1} \widehat{B}_{0} \mathscr{P}_{1} R_{0} \mathscr{P}_{0}, \ldots, i^{n} \widehat{B}_{n-1} \mathscr{P}_{n} \prod_{k=0}^{\overleftarrow{n-1}} R_{k} \mathscr{P}_{k}, \ldots\right)
$$

где $\mathscr{P}_{0}=\mathscr{P}_{1}=I, \mathscr{P}_{k}=e^{i \theta} \mathscr{P}, k \geqslant 2$, и стрелка над матричным произведением указывает направление возрастания индексов матричных сомножителей.

Тогда для ассоциированного с матрищей Якоби $\mathbf{J}_{m}$ оператора $\mathbf{L}_{m}$ справедливы следующие утверждения:

1) первые q столбцов матрицы $\mathbf{P}_{+}$образуют базис в подпространстве $\mathscr{K}_{+}$, а первые р столбцов матрицы $\mathbf{P}_{-}$образуют базис в $\mathscr{K}_{-}$;

2) дефектные числа $m_{+} u m_{-}$ассоциированного оператора $\mathbf{L}_{m}$ coответственно равны q и р.

ЗАмЕчАниЕ 2. Теорема 3 позволяет построить примеры операторов, дефектные числа которых удовлетворяют неравенствам $0 \leqslant m_{+}<m_{-} \leqslant m-1$. Для построения оператора $\mathbf{L}_{m}$, дефектные числа которого удовлетворяют неравенствам $0 \leqslant m_{-}<m_{+} \leqslant m-1$, можно воспользоваться таким простым фактом. Если дефектными числами оператора $\mathbf{L}_{m}$ являются $m_{+}=q, m_{-}=p$, то дефектными числами оператора $(-1) \cdot \mathbf{L}_{m}$ являются $m_{+}=p, m_{-}=q$.

\section{§ 3. Доказательство теорем 2 и 3}

В этом параграфе будут доказаны теоремы 2 и 3. Мы начнем с доказательства теоремы 3 .

ЛЕмма 1. Пусть выполнены условия теоремы 3 и для $m \times m$ блоков матрии, $\mathbf{P}_{ \pm}$введены следующие обозначения:

$$
\left(\mathbf{P}_{+}\right)_{l}=i^{l} \widehat{B}_{l-1} \mathscr{P}_{l} \prod_{k=0}^{\overleftarrow{l-1}} R_{k} \mathscr{P}_{k}, \quad\left(\mathbf{P}_{-}\right)_{l}=(-i)^{l} \widehat{B}_{l-1} \prod_{k=0}^{\overleftarrow{l-1}} R_{k}, \quad l \geqslant 0
$$

Тогда имеют место равенства

$$
\begin{gathered}
A_{0}\left(\mathbf{P}_{ \pm}\right)_{0}+B_{0}\left(\mathbf{P}_{ \pm}\right)_{1}= \pm i\left(\mathbf{P}_{ \pm}\right)_{0} \\
B_{n-1}^{*}\left(\mathbf{P}_{ \pm}\right)_{n-1}+A_{n}\left(\mathbf{P}_{ \pm}\right)_{n}+B_{n}\left(\mathbf{P}_{ \pm}\right)_{n+1}= \pm i\left(\mathbf{P}_{ \pm}\right)_{n}, \quad n \geqslant 1 .
\end{gathered}
$$

ДоказАтельство. Докажем формулы (3.2) для $\mathbf{P}_{+}$. При $n=0$ имеем

$$
A_{0}\left(\mathbf{P}_{+}\right)_{0}+B_{0}\left(\mathbf{P}_{+}\right)_{1}=O \cdot i^{0} R_{0} \mathscr{P}_{0}+I \cdot i^{1} \widehat{B}_{0} \mathscr{P}_{1} R_{0} \mathscr{P}_{0}=i R_{0} \mathscr{P}_{0}=i\left(\mathbf{P}_{+}\right)_{0} .
$$

При $n \geqslant 1$ имеем

$$
\begin{aligned}
& B_{n-1}^{*}\left(\mathbf{P}_{+}\right)_{n-1}+A_{n}\left(\mathbf{P}_{+}\right)_{n}+B_{n}\left(\mathbf{P}_{+}\right)_{n+1} \\
& =i^{n+1} \widehat{B}_{n-1}^{-1}\left(-2\left(I+e^{i \theta} \mathscr{P}\right)^{-1^{*}}-i\left(\mathscr{A}+R_{n} \mathscr{A} R_{n}\right) \mathscr{P}_{n}\right. \\
& \left.\quad+2 R_{n}\left(I+e^{i \theta} \mathscr{P}\right)^{-1} \mathscr{P}_{n+1} R_{n} \mathscr{P}_{n}\right) \prod_{k=0}^{n-1} R_{k} \mathscr{P}_{k}
\end{aligned}
$$




$$
\begin{aligned}
& =i^{n+1} \widehat{B}_{n-1}^{-1}\left(-2\left(I+e^{i \theta} \mathscr{P}\right)^{-1} \mathscr{P}_{n}+\left(I+e^{i \theta} \mathscr{P}\right)^{-1}\left(I-e^{i \theta} \mathscr{P}\right) \mathscr{P}_{n}\right. \\
& +R_{n}\left(I+e^{i \theta \mathscr{P}}\right)^{-1}\left(I-e^{i \theta \mathscr{P}}\right) R_{n} \mathscr{P}_{n} \\
& \left.+2 R_{n}\left(I+e^{i \theta} \mathscr{P}\right)^{-1} \mathscr{P}_{n+1} R_{n} \mathscr{P}_{n}\right) \prod_{k=0}^{\overleftarrow{n-1}} R_{k} \mathscr{P}_{k} \\
& =i^{n+1} \widehat{B}_{n-1}^{-1}\left(\left(I+e^{i \theta} \mathscr{P}\right)^{-1}\left(-2 I+I-e^{i \theta} \mathscr{P}\right)\right. \\
& \left.+R_{n}\left(I+e^{i \theta \mathscr{P}}\right)^{-1}\left(I-e^{i \theta \mathscr{P}}+2 \mathscr{P}_{n+1}\right) R_{n}\right) \mathscr{P}_{n} \prod_{k=0}^{\overleftarrow{n-1}} R_{k} \mathscr{P}_{k} \\
& =i^{n+1} \widehat{B}_{n-1}^{-1}\left(-I+R_{n}^{2}\right) \mathscr{P}_{n} \prod_{k=0}^{\overleftarrow{n-1}} R_{k} \mathscr{P}_{k}=i^{n+1} \widehat{B}_{n-1} \mathscr{P}_{n} \prod_{k=0}^{\overleftarrow{n-1}} R_{k} \mathscr{P}_{k}=i\left(\mathbf{P}_{+}\right)_{n}
\end{aligned}
$$

Здесь первое равенство следует из (2.10) и (2.11), а второе равенство следует из очевидных соотношений

$$
\left(I+e^{i \theta \mathscr{P}}\right)^{-1^{*}}=\left(I+e^{i \theta \mathscr{P}}\right)^{-1} e^{i \theta \mathscr{P}}=\left(I+e^{i \theta \mathscr{P}}\right)^{-1} \mathscr{P}_{n}
$$

Докажем формулы (3.2) для $\mathbf{P}_{-}$. При $n=0$ имеем

$$
A_{0}\left(\mathbf{P}_{-}\right)_{0}+B_{0}\left(\mathbf{P}_{-}\right)_{1}=O \cdot(-i)^{0} R_{0}+I \cdot(-i)^{1} \widehat{B}_{0} R_{0}=-i R_{0}=(-i)\left(\mathbf{P}_{-}\right)_{0} .
$$

При $n \geqslant 1$ имеем

$$
\begin{aligned}
& B_{n-1}^{*}\left(\mathbf{P}_{-}\right)_{n-1}+A_{n}\left(\mathbf{P}_{-}\right)_{n}+B_{n}\left(\mathbf{P}_{-}\right)_{n+1}=(-i)^{n+1} \widehat{B}_{n-1}^{-1}\left(-2\left(I+e^{i \theta} \mathscr{P}\right)^{-1^{*}}\right. \\
& \left.+i\left(\mathscr{A}+R_{n} \mathscr{A} R_{n}\right)+2 R_{n}\left(I+e^{i \theta} \mathscr{P}\right)^{-1} R_{n}\right) \prod_{k=0}^{\overleftarrow{n-1}} R_{k} \\
& =(-i)^{n+1} \widehat{B}_{n-1}^{-1}\left(-2\left(I+e^{i \theta} \mathscr{P}\right)^{-1} e^{i \theta} \mathscr{P}-\left(I+e^{i \theta} \mathscr{P}\right)^{-1}\left(I-e^{i \theta} \mathscr{P}\right)\right. \\
& \left.-R_{n}\left(I+e^{i \theta} \mathscr{P}\right)^{-1}\left(I-e^{i \theta} \mathscr{P}\right) R_{n}+2 R_{n}\left(I+e^{i \theta} \mathscr{P}\right)^{-1} R_{n}\right) \prod_{k=0}^{\overleftarrow{n-1}} R_{k} \\
& =(-i)^{n+1} \widehat{B}_{n-1}^{-1}\left(\left(I+e^{i \theta} \mathscr{P}\right)^{-1}\left(-2 e^{i \theta} \mathscr{P}-I+e^{i \theta \mathscr{P}}\right)\right. \\
& \left.-R_{n}\left(I+e^{i \theta \mathscr{P}}\right)^{-1}\left(I-e^{i \theta \mathscr{P}}-2 I\right) R_{n}\right) \prod_{k=0}^{\overleftarrow{n-1}} R_{k} \\
& =(-i)^{n+1} \widehat{B}_{n-1}^{-1}\left(-I+R_{n}^{2}\right) \prod_{k=0}^{\overleftarrow{n-1}} R_{k}=(-i)^{n+1} \widehat{B}_{n-1} \prod_{k=0}^{\overleftarrow{n-1}} R_{k}=-i\left(\mathbf{P}_{-}\right)_{n}
\end{aligned}
$$

Лемма 1 доказана.

ЗАмечАние 3. Равенства (3.2) будем записывать в следующей компактной форме:

$$
\mathbf{J}_{m} \mathbf{P}_{+}=i \mathbf{P}_{+}, \quad \mathbf{J}_{m} \mathbf{P}_{-}=-i \mathbf{P}_{-} .
$$


Лемма 2. Пусть выполнены условия теоремы 3 и матрицы $\mathbf{P}_{ \pm}$разбить на блоки (3.1). Тогда

$$
\begin{gathered}
\sum_{k=0}^{n}\left(\mathbf{P}_{+}\right)_{k}^{*}\left(\mathbf{P}_{+}\right)_{k}=\left(\prod_{k=0}^{\vec{n}} \mathscr{P}_{k}^{*} R_{k}\right)\left(\prod_{k=0}^{\overleftarrow{n}} R_{k} \mathscr{P}_{k}\right) \\
\sum_{k=0}^{n}\left(\mathbf{P}_{-}\right)_{k}^{*}\left(\mathbf{P}_{-}\right)_{k}=\left(\prod_{k=0}^{\stackrel{n}{n}} R_{k}\right)\left(\prod_{k=0}^{n} R_{k}\right) .
\end{gathered}
$$

ДокАЗАТЕЛЬСтво. При $n=0$ формула (3.4) очевидна. Допустим, что при некотором $l \geqslant 1$ формулы (3.4) доказаны для всех $n \leqslant l$. Тогда для $n=l+1$ имеем

$$
\begin{aligned}
& \sum_{k=0}^{l+1}\left(\mathbf{P}_{+}\right)_{k}^{*}\left(\mathbf{P}_{+}\right)_{k}=\sum_{k=0}^{l}\left(\mathbf{P}_{+}\right)_{k}^{*}\left(\mathbf{P}_{+}\right)_{k}+\left(\mathbf{P}_{+}\right)_{l+1}^{*}\left(\mathbf{P}_{+}\right)_{l+1} \\
& =\prod_{k=0}^{l} \mathscr{P}_{k}^{*} R_{k} \prod_{k=0}^{l} R_{k} \mathscr{P}_{k}+\overleftrightarrow{\prod_{k=0}^{l}} \mathscr{P}_{k}^{*} R_{k} \mathscr{P}_{k+1}^{*} \widehat{B}_{l} \bar{i}^{l+1} i^{l+1} \widehat{B}_{l} \mathscr{P}_{k+1} \prod_{k=0}^{l} R_{k} \mathscr{P}_{k}
\end{aligned}
$$

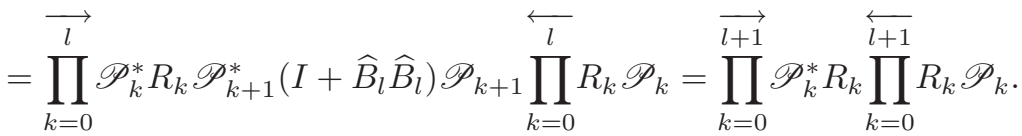

Формулы (3.4) доказаны. Формулы (3.5) доказываются аналогичным образом. Лемма 2 доказана.

Пусть $\mathbf{L}_{m}$ - оператор, ассоциированный с блочной матрицей Якоби $\mathbf{J}_{m}$. Бесконечный столбец

$$
\mathbf{u}=\operatorname{col}\left(u_{0}, u_{1}, u_{2}, \ldots\right), \quad u_{k} \in \mathbb{C}^{m}
$$

(не обязательно принадлежащий $\ell^{2}\left(\mathbb{C}^{m}\right)$ ), называется формальным дефектным вектором оператора $\mathbf{L}_{m}$ в точке $i$ (соответственно $-i$ ), если

$$
\mathbf{J}_{m} \mathbf{u}=i \mathbf{u} \quad\left(\text { соответственно } \mathbf{J}_{m} \mathbf{u}=-i \mathbf{u}\right) .
$$

Множество формальных дефектных векторов в точке $i$ (соответственно $-i$ ) образует линейное пространство, которое мы обозначим через $\mathscr{F}+$ (соответственно через $\mathscr{F}_{-}$).

В условиях теоремы 3 из (3.3) следует, что столбцы бесконечной матрицы $\mathbf{P}_{+}$ (соответственно $\mathbf{P}_{-}$) являются формальными дефектными векторами оператора $\mathbf{L}_{m}$ в точке $i$ (соответственно $-i$ ). Более того, нетрудно убедиться в том, что столбцы матрицы $\mathbf{P}_{+}$образуют базис в пространстве $\mathscr{F}_{+}$, а столбцы матрицы $\mathbf{P}_{-}$образуют базис в пространстве $\mathscr{F}-$. Таким образом, в условиях теоремы 3

$$
\mathscr{F}_{+}=\left\{\mathbf{P}_{+} \varphi: \varphi \in \mathbb{C}^{m}\right\}, \quad \mathscr{F}_{-}=\left\{\mathbf{P}_{-} \varphi: \varphi \in \mathbb{C}^{m}\right\} .
$$

Каждый дефектный вектор (см. (1.2)) является и формальным дефектным вектором. Поэтому имеют место включения $\mathscr{K}_{+} \subset \mathscr{F}_{+}, \mathscr{K}_{-} \subset \mathscr{F}_{-}$. Формальный дефектный вектор является дефектным вектором тогда и только тогда, когда он принадлежит пространству $\ell^{2}\left(\mathbb{C}^{m}\right)$. Таким образом,

$$
\mathscr{K}_{ \pm}=\left\{\mathbf{P}_{ \pm} \varphi: \varphi \in \mathbb{C}^{m}, \mathbf{P}_{ \pm} \varphi \in \ell^{2}\left(\mathbb{C}^{m}\right)\right\} .
$$


Воспользовавшись (3.1), получим

$$
\begin{gathered}
\mathbf{P}_{ \pm}=\operatorname{col}\left(\left(\mathbf{P}_{ \pm}\right)_{0},\left(\mathbf{P}_{ \pm}\right)_{1},\left(\mathbf{P}_{ \pm}\right)_{2}, \ldots\right) \\
\mathbf{P}_{ \pm} \varphi=\operatorname{col}\left(\left(\mathbf{P}_{ \pm}\right)_{0} \varphi,\left(\mathbf{P}_{ \pm}\right)_{1} \varphi,\left(\mathbf{P}_{ \pm}\right)_{2} \varphi, \ldots\right) .
\end{gathered}
$$

Отсюда следует, что вектор $\mathbf{P}_{ \pm} \varphi \in \ell^{2}\left(\mathbb{C}^{m}\right)$ тогда и только тогда, когда существует константа $C>0$ такая, что для всех $n \geqslant 0$ выполняется неравенство

$$
\varphi^{*}\left(\sum_{l=0}^{n}\left(\mathbf{P}_{ \pm}\right)_{l}^{*}\left(\mathbf{P}_{ \pm}\right)_{l}\right) \varphi<C .
$$

Поэтому дефектные пространства $\mathscr{K}_{ \pm}$можно описать так:

$$
\mathscr{K}_{ \pm}=\left\{\mathbf{P}_{ \pm} \varphi: \varphi \in \mathbb{C}^{m}, \varphi^{*}\left(\sum_{l=0}^{n}\left(\mathbf{P}_{ \pm}\right)_{l}^{*}\left(\mathbf{P}_{ \pm}\right)_{l}\right) \varphi<C\right\} .
$$

Отсюда и из леммы 2 имеем

$$
\begin{gathered}
\mathscr{K}_{+}=\left\{\mathbf{P}_{+} \varphi: \varphi \in \mathbb{C}^{m}, \varphi^{*}\left(\prod_{k=0}^{\longrightarrow} \mathscr{P}_{k}^{*} R_{k} \prod_{k=0}^{\overleftarrow{n}} R_{k} \mathscr{P}_{k}\right) \varphi<C\right\}, \\
\mathscr{K}_{-}=\left\{\mathbf{P}_{-} \varphi: \varphi \in \mathbb{C}^{m}, \varphi^{*}\left(\prod_{k=0}^{n} R_{k} \prod_{k=0}^{n} R_{k}\right) \varphi<C\right\} .
\end{gathered}
$$

Лемма 3. Пусть выполненъ условия теоремъ 3. Тогда при $n \geqslant 0$ матричнъе произведения

$$
\overrightarrow{\prod_{k=0}^{n}} \mathscr{P}_{k}^{*} R_{k} \overleftarrow{\prod_{k=0}^{n}} R_{k} \mathscr{P}_{k}, \quad \stackrel{\vec{n}}{\prod_{k=0}} R_{k} \prod_{k=0}^{n} R_{k}
$$

являются диагональными матрицами

$$
\begin{gathered}
\overrightarrow{\prod_{k=0}^{n}} \mathscr{P}_{k}^{*} R_{k} \prod_{k=0}^{\overleftarrow{n}} R_{k} \mathscr{P}_{k}=\operatorname{diag}\left(\gamma_{1}(n), \ldots, \gamma_{q}(n), \gamma_{q+1}(n), \ldots, \gamma_{m}(n)\right), \\
\quad \underset{\prod_{k=0}^{n}}{\longrightarrow} R_{k} \prod_{k=0}^{n} R_{k}=\operatorname{diag}\left(\delta_{1}(n), \ldots, \delta_{p}(n), \delta_{p+1}(n), \ldots, \delta_{m}(n)\right) .
\end{gathered}
$$

Более того, элементы $\gamma_{1}(n), \ldots, \gamma_{q}(n)$ (соответственно элементы $\delta_{1}(n), \ldots$, $\left.\delta_{p}(n)\right)$ положительны и ограничены сверху по $n$, а элементы $\gamma_{q+1}(n), \ldots, \gamma_{m}(n)$ (соответственно элементы $\left.\delta_{p+1}(n), \ldots, \delta_{m}(n)\right)$ положительны и неограничены сверху по $n$.

ДокАзАТЕльство. Сделаем некоторые предварительные замечания. Пусть дана диагональная матрица

$$
\Lambda=\operatorname{diag}\left(\lambda_{1}, \ldots, \lambda_{q}, \lambda_{q+1}, \lambda_{q+2}, \ldots, \lambda_{p}, \lambda_{p+1}, \lambda_{p+2}, \ldots, \lambda_{m}\right)
$$

и матрица $\mathscr{P}$ определена в (2.8). Тогда

$$
\mathscr{P}^{*} \Lambda \mathscr{P}=\operatorname{diag}\left(\lambda_{1}, \ldots, \lambda_{q}, \lambda_{p+1}, \lambda_{q+1}, \ldots, \lambda_{p-1}, \lambda_{p}, \lambda_{p+2}, \ldots, \lambda_{m}\right) .
$$


Итак, матрица $\mathscr{P}^{*} \Lambda \mathscr{P}$ получается из матрицы $\Lambda$ циклической перестановкой диагональных элементов от $(q+1)$-го до $(p+1)$-го.

Теперь исследуем структуру произведения

$$
\overrightarrow{\prod_{k=0}^{n}} \mathscr{P}_{k}^{*} R_{k} \cdot \overleftarrow{\prod_{k=0}^{n}} R_{k} \mathscr{P}_{k}=\mathscr{P}_{0}^{*} R_{0} \cdots\left(\mathscr{P}_{n-1}^{*} R_{n-1}\left(\mathscr{P}_{n}^{*} R_{n}^{2} \mathscr{P}_{n}\right) R_{n-1} \mathscr{P}_{n-1}\right) \cdots R_{0} \mathscr{P}_{0}
$$

Рассмотрим случай, когда $p-q=1$. Из (2.5) имеем

$$
R_{n}^{2}=\operatorname{diag}\left(1+\frac{1}{n^{2}}, \ldots, 1+\frac{1}{n^{2}}, 1+\frac{1}{n^{2}}, 2,2, \ldots, 2\right), \quad n \geqslant 1 .
$$

Отсюда и из равенства $\mathscr{P}_{n}=e^{i \theta} \mathscr{P}, n>1$, следует, что

$$
\mathscr{P}_{n}^{*} R_{n}^{2} \mathscr{P}_{n}=\mathscr{P}^{*} R_{n}^{2} \mathscr{P}=\operatorname{diag}\left(1+\frac{1}{n^{2}}, \ldots, 1+\frac{1}{n^{2}}, 2,1+\frac{1}{n^{2}}, 2, \ldots, 2\right) .
$$

Аналогичным образом,

$$
\begin{gathered}
\mathscr{P}_{n-1}^{*} R_{n-1}\left(\mathscr{P}_{n}^{*} R_{n}^{2} \mathscr{P}_{n}\right) R_{n-1} \mathscr{P}_{n-1}=\operatorname{diag}\left(\prod_{k=n-1}^{n}\left(1+\frac{1}{k^{2}}\right), \ldots, \prod_{k=n-1}^{n}\left(1+\frac{1}{k^{2}}\right)\right. \\
\left.2 \cdot\left(1+\frac{1}{n^{2}}\right),\left(1+\frac{1}{(n-1)^{2}}\right) \cdot 2,2^{2}, \ldots, 2^{2}\right) .
\end{gathered}
$$

Далее,

$$
\begin{aligned}
\mathscr{P}_{n-2}^{*} R_{n-2}\left(\mathscr{P}_{n-1}^{*} R_{n-1}\left(\mathscr{P}_{n}^{*} R_{n}^{2} \mathscr{P}_{n}\right) R_{n-1} \mathscr{P}_{n-1}\right) R_{n-2} \mathscr{P}_{n-2} \\
=\operatorname{diag}\left(\prod_{k=n-2}^{n}\left(1+\frac{1}{k^{2}}\right), \ldots, \prod_{k=n-2}^{n}\left(1+\frac{1}{k^{2}}\right)\right. \\
\left.\quad\left(1+\frac{1}{(n-1)^{2}}\right) \cdot 2^{2},\left(1+\frac{1}{(n-2)^{2}}\right) \cdot 2 \cdot\left(1+\frac{1}{n^{2}}\right), 2^{3}, \ldots, 2^{3}\right) .
\end{aligned}
$$

Наконец,

$$
\begin{aligned}
\mathscr{P}_{n-3}^{*} R_{n-3}\left(\mathscr{P}_{n-2}^{*} R_{n-2}\left(\mathscr{P}_{n-1}^{*} R_{n-1}\left(\mathscr{P}_{n}^{*} R_{n}^{2} \mathscr{P}_{n} R_{n-1} \mathscr{P}_{n-1}\right)\right) R_{n-2} \mathscr{P}_{n-2}\right) R_{n-3} \mathscr{P}_{n-3} \\
=\operatorname{diag}\left(\prod_{k=n-3}^{n}\left(1+\frac{1}{k^{2}}\right), \ldots, \prod_{k=n-3}^{n}\left(1+\frac{1}{k^{2}}\right)\right. \\
\quad\left(1+\frac{1}{(n-2)^{2}}\right) \cdot 2^{2} \cdot\left(1+\frac{1}{n^{2}}\right),\left(1+\frac{1}{(n-3)^{2}}\right)\left(1+\frac{1}{(n-1)^{2}}\right) \cdot 2^{2} \\
\left.2^{4}, \ldots, 2^{4}\right) .
\end{aligned}
$$

Продолжая таким образом, получим, что

$$
\begin{aligned}
\mathscr{P}_{0}^{*} R_{0} & \ldots\left(\mathscr{P}_{n-1}^{*} R_{n-1}\left(\mathscr{P}_{n}^{*} R_{n}^{2} \mathscr{P}_{n}\right) R_{n-1} \mathscr{P}_{n-1}\right) \cdots R_{0} \mathscr{P}_{0} \\
& =\operatorname{diag}\left(\prod_{k=1}^{n}\left(1+\frac{1}{k^{2}}\right), \ldots, \prod_{k=1}^{n}\left(1+\frac{1}{k^{2}}\right), \prod_{k=1}^{n} \alpha_{k, n}^{(1)}, \prod_{k=1}^{n} \alpha_{k, n}^{(2)}, 2^{n}, \ldots, 2^{n}\right) .
\end{aligned}
$$


Здесь при четном $n$ и $1 \leqslant k \leqslant n$

$$
\alpha_{k, n}^{(1)}=\left\{\begin{array}{ll}
2, & \text { если } k \text { четное; } \\
1+\frac{1}{k^{2}}, & \text { если } k \text { нечетное; }
\end{array} \quad \alpha_{k, n}^{(2)}=\left\{\begin{array}{ll}
1+\frac{1}{k^{2}}, & \text { если } k \text { четное; } \\
2, & \text { если } k \text { нечетное; }
\end{array} .\right.\right.
$$

При нечетном $n$ и $1 \leqslant k \leqslant n$

$$
\alpha_{k, n}^{(1)}=\left\{\begin{array}{ll}
1+\frac{1}{k^{2}}, & \text { если } k \text { четное; } \\
2, & \text { если } k \text { нечетное; }
\end{array} \quad \alpha_{k, n}^{(2)}= \begin{cases}2, & \text { если } k \text { четное; } \\
1+\frac{1}{k^{2}}, & \text { если } k \text { нечетное. }\end{cases}\right.
$$

Отсюда и из сходимости произведения $\prod_{k=1}^{\infty}\left(1+1 / k^{2}\right)$ следует, что

$$
\begin{aligned}
\mathscr{P}_{0}^{*} R_{0} \cdots\left(\mathscr{P}_{n-1}^{*} R_{n-1}\left(\mathscr{P}_{n}^{*} R_{n}^{2} \mathscr{P}_{n}\right) R_{n-1} \mathscr{P}_{n-1}\right) \cdots R_{0} \mathscr{P}_{0} \\
=\operatorname{diag}(\underbrace{\prod_{k=1}^{n}\left(1+\frac{1}{k^{2}}\right), \ldots, \prod_{k=1}^{n}\left(1+\frac{1}{k^{2}}\right)}_{\text {ограничены по } n}, \underbrace{\left.\prod_{k=1}^{n} \alpha_{k, n}^{(1)}, \prod_{k=1}^{n} \alpha_{k, n}^{(2)}, 2^{n}, \ldots, 2^{n}\right)}_{\text {неограничены по } n} .
\end{aligned}
$$

Таким образом, доказаны утверждения леммы 3 относительно произведения (3.8) при условии $p-q=1$. При условии $p-q>1$ доказательство проводится аналогичным образом. Утверждения леммы 3 относительно произведения (3.9) следуют из (3.7) и очевидного равенства

$$
\overrightarrow{\prod_{k=0}^{n}} R_{k} \overleftarrow{\prod_{k=0}^{n}} R_{k}=\prod_{k=0}^{n} R_{k}^{2}=\operatorname{diag}\left(\prod_{k=1}^{n}\left(1+\frac{1}{k^{2}}\right), \ldots, \prod_{k=1}^{n}\left(1+\frac{1}{k^{2}}\right), 2^{n}, \ldots, 2^{n}\right)
$$

Лемма 3 доказана.

Лемма 4. Пусть выполнены условия теоремы 3. Для $s=p$ или $s=q$ обозначим $\mathbb{C}_{s}^{m}=\left\{\operatorname{col}\left(\varphi_{1}, \ldots, \varphi_{s}, 0, \ldots, 0\right) \in \mathbb{C}^{m}\right\}$. Тогда

$$
\mathbf{P}_{+} \varphi \in \mathscr{K}_{+} \Longleftrightarrow \varphi \in \mathbb{C}_{q}^{m}, \quad \mathbf{P}_{-} \varphi \in \mathscr{K}_{-} \Longleftrightarrow \varphi \in \mathbb{C}_{p}^{m} .
$$

ДокАзАтельство. Сначала докажем первое из равенств (3.10). Пусть вектор $\mathbf{P}_{+} \varphi \in \mathscr{K}_{+}$. С учетом (3.8) имеем

$$
\begin{gathered}
\varphi^{*}\left(\prod_{k=0}^{\vec{n}} \mathscr{P}_{k}^{*} R_{k} \prod_{k=0}^{\overleftarrow{n}} R_{k} \mathscr{P}_{k}\right) \varphi=\varphi^{*} \operatorname{diag}\left(\gamma_{1}(n), \ldots, \gamma_{q}(n), \gamma_{q+1}(n), \ldots, \gamma_{m}(n)\right) \varphi \\
\quad=\left|\varphi_{1}\right|^{2} \gamma_{1}(n)+\cdots+\left|\varphi_{q}\right|^{2} \gamma_{q}(n)+\left|\varphi_{q+1}\right|^{2} \gamma_{q+1}(n)+\cdots+\left|\varphi_{m}\right|^{2} \gamma_{m}(n) .
\end{gathered}
$$

В силу (3.6) получаем, что

$$
\left|\varphi_{1}\right|^{2} \gamma_{1}(n)+\cdots+\left|\varphi_{q}\right|^{2} \gamma_{q}(n)+\left|\varphi_{q+1}\right|^{2} \gamma_{q+1}(n)+\cdots+\left|\varphi_{m}\right|^{2} \gamma_{m}(n)<C .
$$

Отсюда и из леммы 3 следует, что $\varphi_{q+1}=\cdots=\varphi_{m}=0$, т.е. $\varphi \in \mathbb{C}_{q}^{m}$.

Пусть теперь $\varphi \in \mathbb{C}_{p}^{m}$. По лемме 3 имеем

$$
\varphi^{*}\left(\prod_{k=0}^{\vec{n}} R_{k} \overleftarrow{\prod_{k=0}^{n}} R_{k}\right) \varphi=\left|\varphi_{1}\right|^{2} \gamma_{1}(n)+\cdots+\left|\varphi_{q}\right|^{2} \gamma_{q}(n)<C
$$

Отсюда и из (3.6) следует, что $\mathbf{P}_{+} \varphi \in \mathscr{K}_{+}$и первое равенство (3.10) доказано. Аналогичным образом доказывается второе равенство (3.10). Лемма 4 доказана. 
ДоКАЗАТЕЛЬСТВо теОРемЫ 3. Пусть выполнены условия теоремы 3. Тогда первое утверждение теоремы 3 немедленно следует из леммы 4. Второе утверждение теоремы 3 является очевидным следствием первого.

ЗАмечАниЕ 4. Теорему 2 можно рассматривать как вырожденный случай теоремы 3. А именно, если в формулировке и доказательстве теоремы 3 положить $\mathscr{P}=I, \theta=0$ (см. (2.8) и (2.9)) и сделать очевидные упрощения, то получим формулировку и доказательство теоремы 2.

\section{Список литературы}

[1] М.Г. Крейн, "Бесконечные $J$ матрицы и матричная проблема моментов", Докл. AH CCCP, 69:3 (1949), 125-128.

[2] М. Г. Крейн, "Основные положения теории представления эрмитовых операторов с индексом дефекта $(m, m)$ ", Укр. матем. журн., 1:2 (1949), 3-66; англ. пер.: M. G. Krein, "Fundamental aspects of the representation theory of Hermitian operators with deficiency index (m,m)", Amer. Math. Soc. Transl. 2, 97 (1971), 75-143.

[3] Ю.М. Березанский, Разложение по собственным функииям самосопряженных операторов, Наукова думка, Киев, 1965; англ. пер.: Yu. M. Berezans'kiі̌, Expansions in eigenfunctions of selfadjoint operators, Amer. Math. Soc., Providence, RI, 1968.

[4] В.И. Коган, "Об операторах, порожденных $l_{p}$-матрицами в случае максимальных индексов дефекта", Теория функиий, функи. анализ и их прил., 1970, № 11, 103-107.

[5] А.Г. Костюченко, К.А. Мирзоев, "Признаки вполне неопределенности якобиевых матриц с матричными элементами", Функи. анализ и его прил., 35:4 (2001), 32-37; англ. пер.: A. G. Kostyuchenko, K. A. Mirzoev, "Complete indefiniteness tests for Jacobi matrices with matrix entries", Funct. Anal. Appl., 35:4 (2001), 265-269.

[6] Ю.М. Дюкарев, "О дефектных числах симметрических операторов, порожденных блочными матрицами Якоби", Матем. сб., 197:8 (2006), 73-100; англ. пер.: Yu. M. Dukarev, "Deficiency numbers of symmetric operators generated by block Jacobi matrices", Sb. Math., 197:8 (2006), 1177-1203.

[7] И. В. Ковалишина, В.П. Потапов, "Радиусы круга Вейля в матричной задаче Неванлинны-Пика", Теория операторов в функиионалъных пространствах и ее приложения, Наукова думка, Киев, 1981, 25-49.

Ю. М. Дюкарев (Yu. M. Dyukarev)

Харьковский национальный университет им. В.Н.Каразина, Украина

E-mail: Yury.M.Dyukarev@univer.kharkov.ua
Поступила в редакцию 24.08.2009 и 11.04.2010 